\title{
Caroli's Disease
}

\section{Col NK Biswas*, Col U Raju+, Lt Col K Sodhi"}

MJAFI 2007; 63 : 388-389

Key Words : Biliary duct dilatation; Cholangitis; Hepatomegaly

\section{Introduction}

$\mathrm{C}$ aroli's disease is a rare congenital disorder of the liver characterized by saccular or fusiform dilatation of the intrahepatic biliary ducts. In majority the transmission is autosomal recessive [1] but autosomal dominant inheritance has also been reported [2]. The main clinical features are intermittent abdominal pain, hepatomegaly and recurrent cholangitis $[1,3]$. We report an unusual case where in spite of advanced cystic dilations of biliary tree, the patient did not suffer a single episode of cholangitis.

\section{Case Report}

A 10 year old male child presented with the complaints of intermittent right upper quadrant abdominal pain of four years duration. There was no history of fever, jaundice, haematemesis or melena. There was no other significant past history. He was the first child of a third degree consanguineous marriage, the other two siblings being healthy. Physical examination revealed an averagely built and nourished child with no pallor, icterus or significant

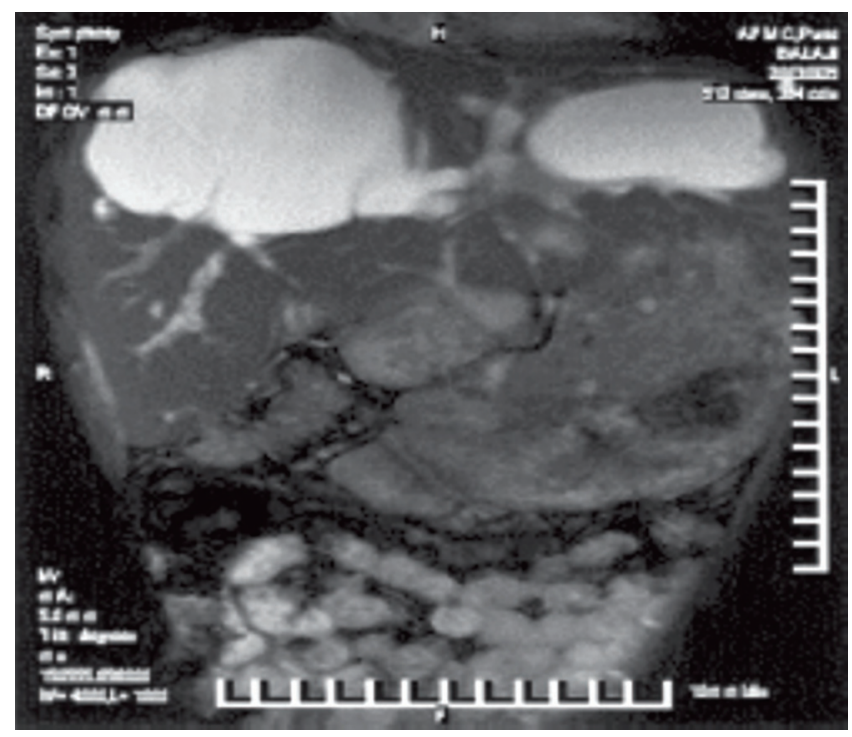

Fig. 1: MRI scan of liver showing multiple cystic lesions in both lobes of liver. lymphadenopathy. The liver was enlarged, $13 \mathrm{~cm}$ below right costal margin in the midclavicular line. It was firm in consistency and non tender. The spleen was not palpable. Other systemic examination was not contributory. Laboratory examination revealed a normal haemogram. Liver function tests viz serum bilirubin, serum albumin, prothrombin time, alanine aminotransferase (ALT), aspartate aminotransferase (AST) and alkaline phosphatase were within normal limits. Renal function tests and chest radiograph were non contributory. Ultrasonography (USG) of abdomen showed multiple cystic lesions within the liver occupying both the lobes but more pronounced in the right lobe. Both kidneys were normal. Upper gastrointestinal (UGI) endoscopy did not reveal any abnormality. Magnetic resonance imaging(MRI) of abdomen (Fig.1), showed multiple large cysts within the liver occupying both the lobes but the continuity of the cysts with biliary tree could not be clearly delineated. Thereafter hepatic scintigraphy using Technetium-99 with meprofenin was carried out. Images were taken intermittently for 24 hours. The image taken at 30 minutes showed two large photopenic areas in the liver (Fig. 2), which were filled up due to pooling of bile in the 24 hours image, signifying

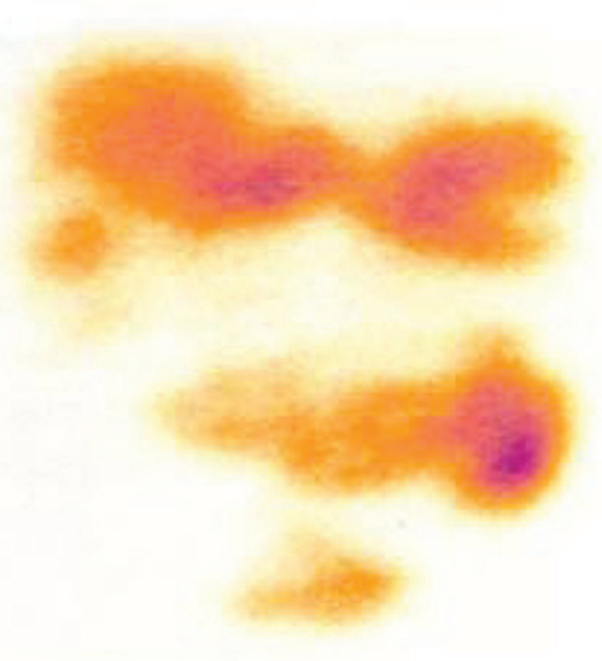

Fig. 2: Hepatic scintigraphy 24 hour image showing pooling of bile in the cystic lesions.

"Classified Specialist (Paediatrics), Military Hospital, Jabalpur. ${ }^{+}$Senior Advisor (Paediatrics \& Neonatology), ${ }^{*}$ Classified Specialist (Paediatrics), Command Hospital (Southern Command) Pune.

Received : 24.11.2005; Accepted :10.04.2007 
communication of the cysts with the intra hepatic biliary tree. Since there was no evidence of hepatic fibrosis on USG and MRI, liver biopsy was not done. A diagnosis of isolated Caroli's disease was made and the child was treated conservatively. Partial lobectomy was advised but the parents did not consent for the same. The parents were counselled and the child is symptom free till date, on regular follow up.

\section{Discussion}

1n 1958 Jacques Caroli first described this rare congenital anomaly of intra hepatic bile ducts of which he described two variants [4].The first variant called Caroli's syndrome is associated with hepatic fibrosis and portal hypertension. The second variant called Caroli's disease is associated with autosomal recessive polycystic kidney disease, autosomal dominant polycystic kidney disease and choledochal cysts [4,5].The cystic dilatations in Caroli's disease may be localized to one lobe of liver or involve the whole organ. It results from an arrest in ductal remodeling of large intra hepatic bile ducts [1].

In children most cases are diagnosed between 2-16 years of age with a peak incidence at 10 years[3].The clinical features are intermittent abdominal pain and hepatomegaly. Recurrent bouts of cholangitis, abscess due to bile stasis, septicemia, stone formation within cysts and cholangiocarcinoma are common complications. Acute obstructive episodes are characterized by fever, pruritus, jaundice, tender hepatomegaly with modest elevations of serum bilirubin, aminotransferases and alkaline phosphatase $[1,3,6]$. In our case in spite of the advanced cystic dilatations, there was no history suggestive of cholangitis or abscess formation .

The current diagnostic imaging modalities demonstrate cystic dilatations of large proximal intra hepatic bile ducts interspersed with normal ducts. The cystic lesions are usually saccular or fusiform. They are commonly multifocal and involve both lobes of liver as in our case. Rarely the lesions may be segmental or localized to a single lobe of liver [7]. The diagnosis rests on demonstrating that the cysts are in continuity with the intra hepatic biliary tree. In our case hepatic scintigraphy demonstrated the origin of cysts from intra hepatic bile ducts and confirmed the diagnosis.

The treatment of Caroli's disease depends on the presentation and location of the cysts. Appropriate antibiotic therapy is required when infection occurs in the form of cholangitis, liver abscess and septicemia. In case of intrahepatic stone formation apart from surgery, treatment with ursodeoxycholic acid is indicated [8]. When the disease is localized to a single lobe, lobectomy can not only cure but also reduce the risk of subsequent occurrence of cholangiocarcinoma [9]. When the lesions are diffuse involving both the lobes, the prognosis is guarded due to difficulties in controlling cholangitis and biliary lithiasis and a significant risk of developing malignancy. The treatment is conservative and liver transplantation is done in selected cases [10].

\section{Conflicts of Interest}

None identified

\section{References}

1. Suchy FJ. Cystic diseases of the biliary tract and liver. In: Behrman RE, Kliegman RM, Jenson HB, editors. Nelson Textbook of Pediatrics. $17^{\text {th }}$ ed. Philadelphia : WB Saunders, 2004; 1343-4.

2. Tsuchida Y, Sato T, Sanjo K. Evaluation of long term results of Caroli's disease: 21 years'observation of a family with autosomal dominant inheritence, and review of the literature. Hepatosplenomegaly 1995;42:175-81.

3. De Tommaso AM, Santos DS, Hessel G. Caroli's disease : 6 case studies. Acta Gastroenterologica Latinoamericana 2003;33:47-51.

4. Baslul K. Caroli's Disease. Indian Pediatrics 2004;41:848-50.

5. Jordan D, Harpaz N, Thung SN. Caroli's disease and adult polycystic kidney disease: a rarely recognized association. Liver 1989;9:30-5.

6. Faria G, Flores P, Burgos L, et al. Primary cholangiocacinoma associated with Caroli's disease. Revista Medica de Chile 2001;129: 1433-8.

7. Levy AD, Rohrmann CA Jr, Murakata LA, Lonergan GJ. Caroli's disease:radiologic spectrum with pathologic correlation. Am J Roentgenol 2002;179:1053-7.

8. Ros E, Navarro S, Bru C, Gilabert R, Bianchi L, Bruguera M. Ursodeoxycholic acid treatment of primary hepatolithiasis in Caroli's syndrome. Lancet 1993; 342:404-6.

9. Espinoza R, San Martin S, Court F, Vera E, Ferreira R, Croxatto H. Hepatic resection in localized Caroli's disease. Revista Medica de Chile 2003;131:183-9.

10. Waechter FL, Sampaio JA, Pinto RD, Alvares-da-Silva MR, Cardoso FG, Francisconi C, et al.The role of liver transplantation in patients with Caroli's disease. Hepato-Gastroenterology 2001;48:672-4. 